\title{
Sensor-Based Detection Approach for Passenger Flow Safety in Chinese High-speed Railway Transport Hub
}

\author{
Xie Zhengyu \\ School of Traffic and Transportation \\ Beijing Jiaotong University \\ Beijing, China \\ xiezhy2001@gmail.com
}

\author{
Qin Yong \\ State Key Laboratory of Rail Traffic Control and Safety \\ Beijing Jiaotong University \\ Beijing, China \\ qingyong2146@126.com
}

\begin{abstract}
Passenger flow safety detection in high-speed railway transport hub is considered in this paper. For accurately detecting the passenger flow safety, an improved watershed algorithm and a recognition algorithm are respectively proposed in sensor-based detection process. Computational experiments on sensor data from a specific Chinese high-speed railway transport hub show that the proposed algorithms are effective and efficient for detecting the passenger flow safety in high-speed railway transport hub.
\end{abstract}

Keywords-intelligent transportation system; sensor-based detection; passenger flow safety; high-speed railway transport hub

\section{INTRODUCTION}

Over the past seven-year period, a huge high-speed railway network has been built in China, with lines in operation amounting to $11,132 \mathrm{~km}$. The rapid developing of high-speed railway leads the passenger distribution quantity increasing sharply in transport hubs, which make the safety management of hub confront with serious challenges. Current video surveillance systems in high-speed railway transport hubs are relatively simple, which cannot automatically detect the potential safety hazards in high density passenger flow, only can passively respond for the emergency. According to the stringent safety detection demands, the detection method of video surveillance systems needs become more intelligence and automatic.

Intelligent video surveillance is an important content in computer vision study, which uses computer vision technology and artificial intelligence technology to analyze, process and describe the image sequences of video surveillance. The powerful processing ability of computer can rapidly filter out interferential information, extract objective information, and achieve the detecting, locating and tracking of surveillance objectives. Currently, the intelligent video surveillance is widely applied in transportation fields including: moving people and vehicles detecting and tracking [1-3], abnormal behavior detecting [4-6], vehicle shape, color and plate number recognition [7,8], sensitive area intrusion detection $[9,10]$, unusual crowd detection [11,12], etc. With more video surveillance sensors used in transport hubs, the studies focus on the moving object detection in hubs become a new hotspot. However, in surveillance scenes, the interference of weather, illumination, shadow and repetitive motion of passengers make video surveillance difficult to detect objects fast and reliably.

The passenger flow safety detection in high-speed railway transport hub belongs to the moving object detection problem, which has been investigated in many literatures. The universal process of moving object detection, and all typical algorithms and their advantages were expounded, then summarized algorithms characteristics, and compared the performances of some algorithms. Finally, pointed out key issues and directions of future study in this area [13]. According to comparing moving object detection methods and typical background modeling methods, a suitable method was selected for background modeling in high-speed railway transport hub [14]. An efficient adaptive segmentation algorithm was developed for color video surveillance sequence in real time with nonstationary background [15]. A hybrid temporal-spatio forecasting approach was proposed to obtain the passenger flow safety in high-speed railway transport hub .The approach combined temporal forecasting based on radial basis function neural network and spatio forecasting based on spatial correlation degree [16].

According to the literature review above, several moving object detection approaches were present. However, there is no universal method can solve different problems. Detection method must be developed based on the detection objects and applied places. For passenger flow detection, a background model based on Dempster-Shafer theory and a recognition algorithm based on connected domain were developed [18]. In this paper, based on the detection method developed in Ref [18], we add an image segmentation algorithm in sensor-based detection process and propose an improved recognition algorithm to improve the accuracy of passenger flow safety detection in high-speed railway transport hub. 
The rest of paper is organized as follows. Section 2 introduces the process of passenger flow safety detection based on surveillance sensors. Section 3 proposes an improved watershed algorithm based on tag obtained automatically. A recognition algorithm based on connected domain characters is developed in section 4. Section 5 takes experiments on sensor data from a specific Chinese high-speed railway transport hub to verify the algorithms we proposed. Section 6 concludes the paper and provides the direction for future work.

\section{PASSENGER FLOW SAFETY DETECTION PROCESS BASED ON SURVEILLANCE SENSORS}

According to the basic process of moving object detection, the passenger flow safety detection process based on surveillance sensors in high-speed railway transport hub is designed as follows.

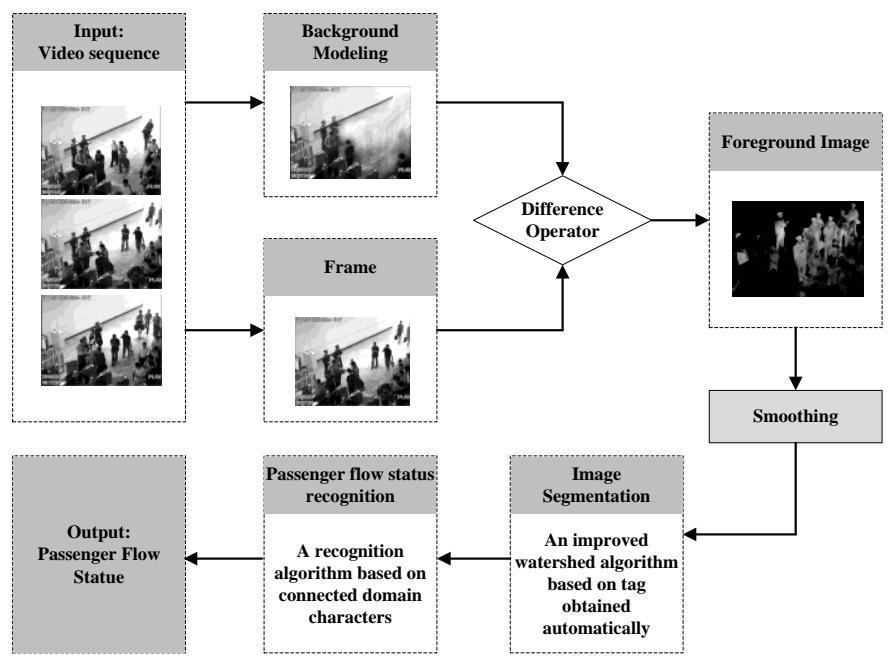

Figure 1. Passenger flow safety detection process based on surveillance sensors.

(1) Input and output: the process input is the video sequence obtained from video surveillance sensors in highspeed railway transport hub, and the output is the passenger flow status of detection areas.

(2) Background modeling and updating: according to the characteristics of detection areas in high-speed railway transport hub, a background model is developed to build a reliable background image, and an updating strategy is proposed to update the background image for adapting the dynamic circumstance change in hub.

(3) Image segmentation: based on the characteristics of passenger flow foreground images, an image segmentation algorithm is proposed to accurately filter out interferential information and extract object information.

(4) Passenger flow safety recognition: a passenger flow safety recognition method is proposed to recognize the passenger flow safety from the extract object information which is obtained by image segmentation.

In the process mentioned in Fig.1, the background modeling and updating have been studied [17]. In this paper, we consider the image segmentation algorithm and passenger flow status recognition method. Firstly, an improved watershed algorithm based on tag obtained automatically is proposed to avoid excessive segmentation and reduce useful information losing. Then, for solving severe overlap in passenger flow image, a recognition algorithm based on connected domain characters is developed. The two algorithms are detailed in the following sections.

\section{AN IMPROVED WATERSHED ALGORITHM BASED ON TAG OBTAINED AUTOMATICALLY}

In order to meet high segmentation accuracy demands of high-speed railway transport hub, the image segmentation algorithm need to reduce useful information losing, and extract the passenger flow information in the segmentation process. As a classical image segmentation method, the watershed algorithm has the advantages of edge location accuracy, simple operation, etc, and has been applied in many fields. However, traditional watershed algorithm has disadvantage of excessive segmentation, which seriously interferes the recognition of object edge. For avoiding the excessive segmentation, several improved algorithms are proposed. The improved watershed algorithm based on tag is one of improved algorithms, improved from the segmentation theory of watershed algorithm, and essentially solve the excessive segmentation. So we select this algorithm as segmentation algorithm.

In typical tag extracting method, threshold is determined by subjectively manual setting, which is suitable for small quantity image processing. But the setting method cannot meet the timeliness requirement of video surveillance in high-speed railway transport hub, and has low adaptation for the dynamic circumstance change. So according to the demands of segmentation in high-speed railway transport hub, we propose an improved watershed algorithm based on tag obtained automatically. The algorithm firstly sorts the gradient of each pixel in the image, tags the threshold by using the image gray information, sets the tag value as the minimum image gradient, and modifies the gradient image. Then, use the immersion to process the modified gradient image. The algorithm process is shown in Fig.2.

Main steps of the algorithm are described as follows.

Step 1: calculate the gradient of each pixel in the image, and scan the whole image to get the probability density $P_{i}$ of each gradient. The position of each pixel in sorted array is determined by the calculation of graded distribution cumulative probability and the gradient of each pixel. In the sorted array, the pixel with the low gradient value is put in the front of array.

Step 2: process the pixels in accordance with the sequence from low to high. The pixels having same gradient are processed as one gradient hierarchy.

Step 3: count the maximum gradient set $U_{\max }$ and minimum gradient set $U_{\min }$, calculate the average gradient $h_{\max }$ of the maximum gradient set and the average gradient $h_{\min }$ of the minimum gradient set. Then count the secondary minimum gradient set $U_{\min }$ 'from all pixels whose gradient is greater than $h_{\min }$, and calculate the average gradient $h_{\min }{ }^{\prime}$ of the secondary 
minimum gradient set. Based on the data mentioned above, the threshold $T$ is calculated as follows:

$$
T=h_{\min }+h_{\min }{ }^{\prime}-\sigma
$$

Where $\sigma$ is the mean square error of $U_{\text {max }}$.

Step 4: according to the threshold obtained in step 3, tag the minimum gradient, and sort the pixels whose gradients are lower than $T$ into one gradient hierarchy.

Step 5: process the $h$ gradient hierarchy, putting all pixels whose neighborhoods have been tagged into a first-in first-out (FIFO) queue.

Step 6: if the queue is empty, go to step 8. Otherwise, go to step 7.

Step 7: process the neighborhoods of the first pixels in the queue. If the neighborhood of pixel has been tagged, updates the pixel tag based on the neighborhood tag, and then delete this pixel from the queue, go to step 6. If the neighborhood of pixel has not been tagged, put this neighborhood into the FIFO queue, update the queue, go to step 6 .

Step 8: scan the $h$ gradient hierarchy to check if any pixel is not tagged. If there is a pixel not tagged, add 1 to the current tag value, and take this value as the tag value of the untagged pixel, go to step 4. Otherwise, go to step 9.

Step 9: set $h=h+1$, if $h \leq$ max_hierarchy, then go to step 4 . Otherwise, segmentation finishes.

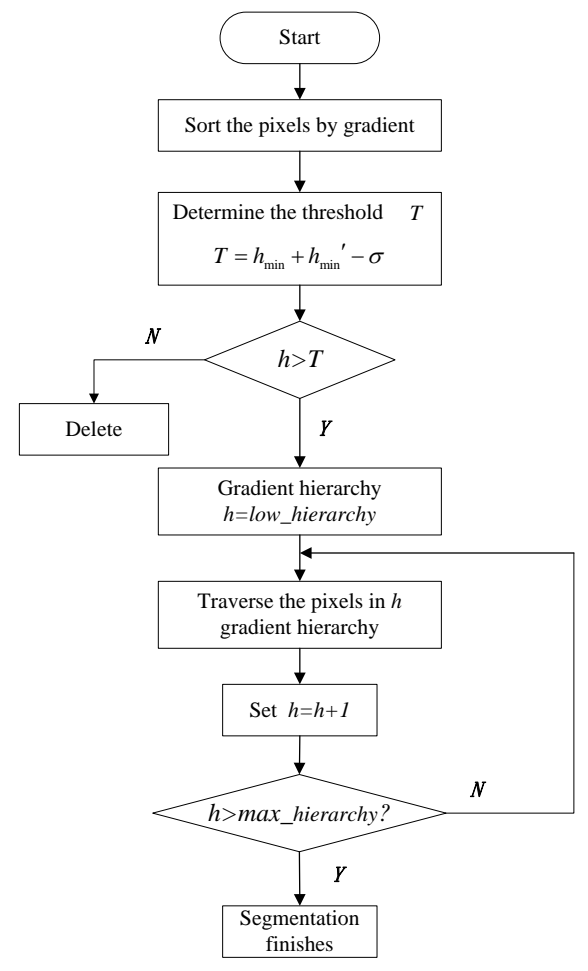

Figure 2. Improved watershed algorithm process

\section{A RECOGNITION ALGORITHM BASED ON CONNECTED DOMAIN CHARACTERS}

In ideal conditions, the passenger objects should be segmented in different connected domains based on the previous processing. According to the amount of the connected domains, the passenger quantity can be calculated, and the other passenger status could be obtained by passenger quantity. But in practical application, the crowd in passenger flow in high-speed railway transport hub makes passenger objects overlapped in the passenger flow image, and several objects may belong to one connected domain. Simply using the connected domains to count the passenger quantity can cause serious error. According to the analysis of passenger flow images in high-speed railway transport hub, we can classify the connected domains of passenger flow images after segmentation into two types. The first type is the large noncircular connected domains which are caused by passengers overlapped. The other type is the small connected domains which are generated by independent passenger. Based on the analysis and classification of connected domains in high-speed railway transport hub, we propose a recognition algorithm based on connected domain characters to accurately recognize the passenger flow safety in high-speed railway transport hub.

The recognition algorithm firstly classifies all the connected domains into two parts. For the independent passenger connected domains, directly calculate the passenger amount. Then, for the passengers overlapped connected domains, calculate the passenger amount by quasi-circle analyzing and matching. Finally, sum the passenger amount of two parts, and obtain the total passenger quantity in the surveillance image.

The notations of algorithm are defined as follows. $n$ is amount of connected domains. $i$ is the number of connected domains. $S_{i}$ is the area of the $i$ connected domain. $L_{i}$ is the circumference of the $i$ connected domain. $M_{i}$ is the measurement of the $i$ connected domain,. $D_{s}, D_{l}, D_{m}$ are thresholds, $D_{s}>D_{l} \cdot n_{i 1}, n_{i 2}$ are passenger amount of two types connected domains. $n_{i}$ is the passenger amount of the $i$ connected domain. $n_{1}$ is the amount of quasi-circulars. $N$ is the passenger amount in the image. The algorithm process is shown in Fig.3.

Main steps of the algorithm are described as follows.

Step 1: mark all the connected domains by the sequential algorithm, go to step 2 .

Step 2: calculate the area of the $i$ connected domain $S_{i}$ by Eq.2

$$
S_{i}=\left(B\left(C_{i}\right)-T\left(C_{i}\right)\right) \times\left(R\left(C_{i}\right)-L\left(C_{i}\right)\right)
$$

Where $T\left(C_{i}\right)$ is the top boundary value of the $i$ connected domain, $B\left(C_{i}\right)$ is the bottom boundary value of the $i$ connected domain, $L\left(C_{i}\right)$ is the left boundary value of 
the $i$ connected domain, $R\left(C_{i}\right)$ is the right boundary value of the $i$ connected domain, and $T\left(C_{i}\right)<B\left(C_{i}\right), L\left(C_{i}\right)<R\left(C_{i}\right)$.

Step 3: compare $S_{i}$ and $D_{s}$, if $S_{i}<D_{s}$, eliminate this connected domain, let $i=i+1$, then go to step 7 . Otherwise, go to step 4 .

Step 4: compare $S_{i}$ and $D_{l}$, if $S_{i}<D_{l}$, let $n_{i 1}=0$, calculate the circumference of the $i$ connected domain $L_{i}$, then calculate the measurement of the $i$ connected domain $M_{i}$ by Eq.3, then go to step 5. Otherwise, go to step 6.

$$
M_{i}=4 \pi \cdot S_{i} / L_{i}^{2}
$$

Step 5: compare $M_{i}$ and $D_{m}$, if $M_{i}>D_{m}$, let $n_{i 1}=1$, $n_{i}=n_{i 1}$ and $i=i+1$, then go to step 7. Otherwise, eliminate this connected domain, let $i=i+1$, then go to step 7 .

Step 6: let $n_{i 2}=0$, and calculate the amount of quasicirculars $n_{1}$. Then, let $n_{i 2}=n_{1}, n_{i}=n_{i 2}$ and $i=i+1$, then go to step 7 .

Step 7: compare $i$ and $n$, if $i>n$, calculate the $N$ by Eq.4, and output the passenger flow safety, the algorithm finish. Otherwise, go to step 3.

$$
N=\sum_{i=1}^{n} n_{i}
$$

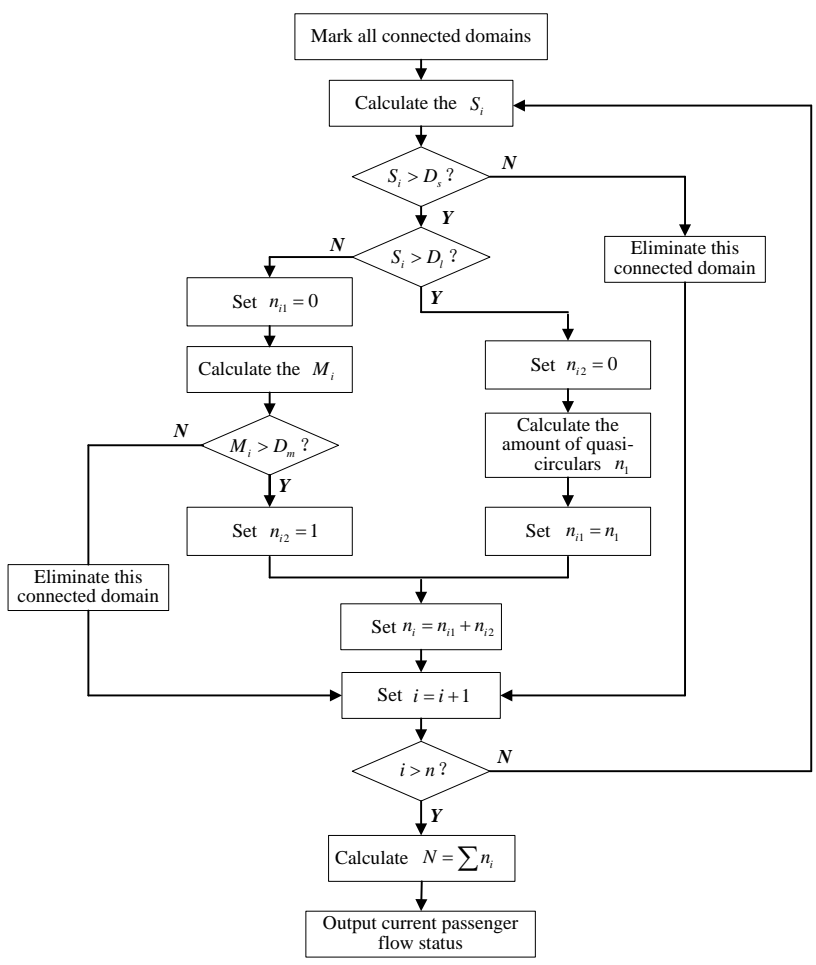

Figure 3. Recognition algorithm process

\section{COMPUTATIONAL EXPERIMENTS}

In this section, computational experiments are conducted to illustrate the detection algorithms proposed above. Actual sensor data from a specific Chinese high-speed railway transport hub surveillance video sequence is taken as an example. Fig. 4 is a sample of surveillance video sequence from a sensor.

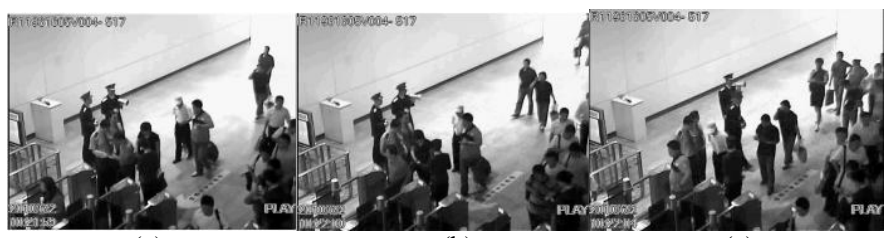

(a)

(b)

(c)

Figure 4. A sample of surveillance video sequence. (a) the 1st frame image, (b) the 40th frame image, (c) the 100th frame image

A background image was built by the background model [17]. Then we used difference operators between current frame and background image to get the foreground image. The current frame, background image and foreground image are shown in Fig.5.

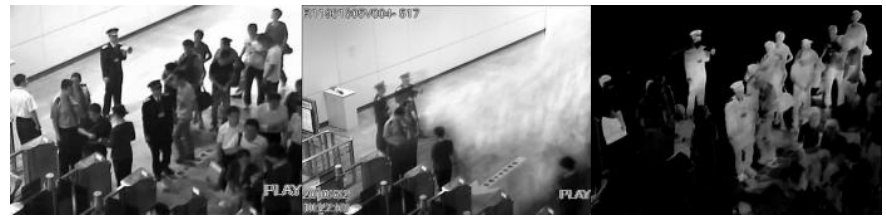

(a)

(b)

(c)

Figure 5. Current frame, background image and foreground image. (a) current frame, (b) background image, (c) foreground image

In order to reduce noise signal impact and improve the resolution of objects in foreground image, a smoothing was applied before image segmentation. We choose mean filter and median filter as smoothing methods, and make a comparison between them. The comparison is shown in Fig.6.

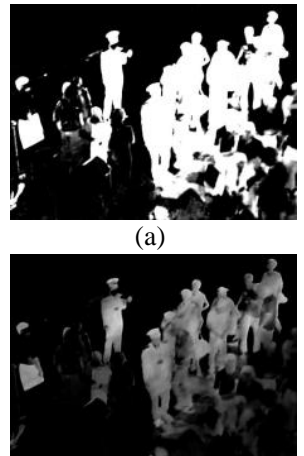

(d)

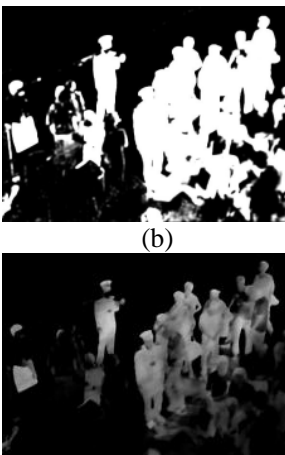

(e)

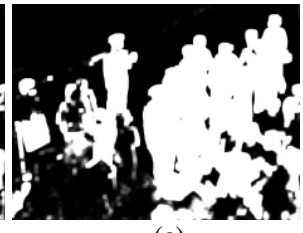

(c)

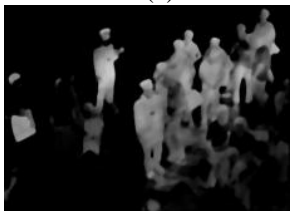

(f)
Figure 6. Comparison between mean filter and median filter. (a) $3 * 3$ mean filter, (b) $5 * 5$ mean filter, (c) $9 * 9$ mean filter, (d) $3 * 3$ median filter, (e) $5 * 5$ median filter, (f) $9 * 9$ median filter

As observed in Fig.6, the performance of the median filter is better than the mean filter. So we chose median filter for the foreground image smoothing. After foreground image smoothing, we used the improved watershed algorithm mentioned in section 3 for foreground image segmentation. The segmentation process is shown in Fig.7. 


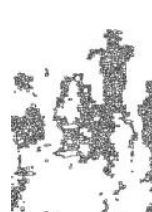

(a)

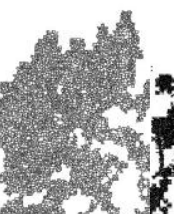

)

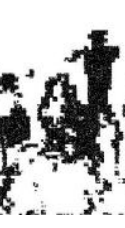

(b)

Figure 7. Segmentation process

After the foreground image segmentation, we applied the passenger flow status recognition algorithm mentioned in section 4 to recognize the passenger amount in the surveillance video. The comparison between actual passenger amount and recognition amount is shown in Fig.8, and the recognition accuracy comparison between our methods (OM) and method mentioned in Ref [18] is shown in Fig.9.

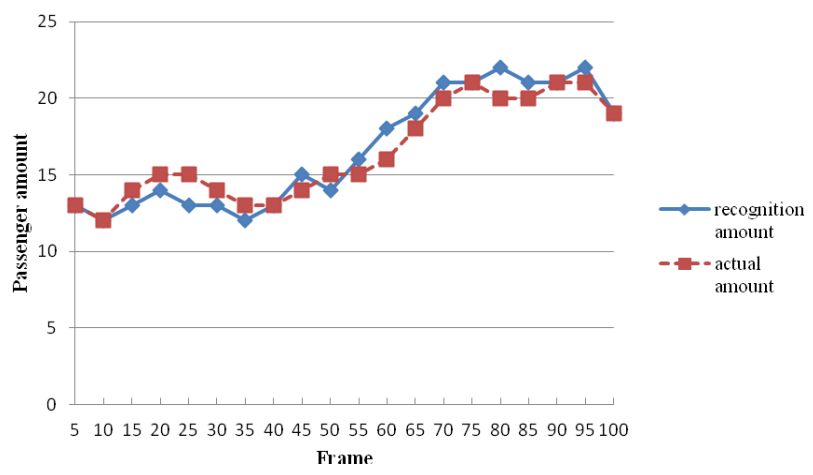

Figure 8. Comparison between actual passenger amount and recognition amount

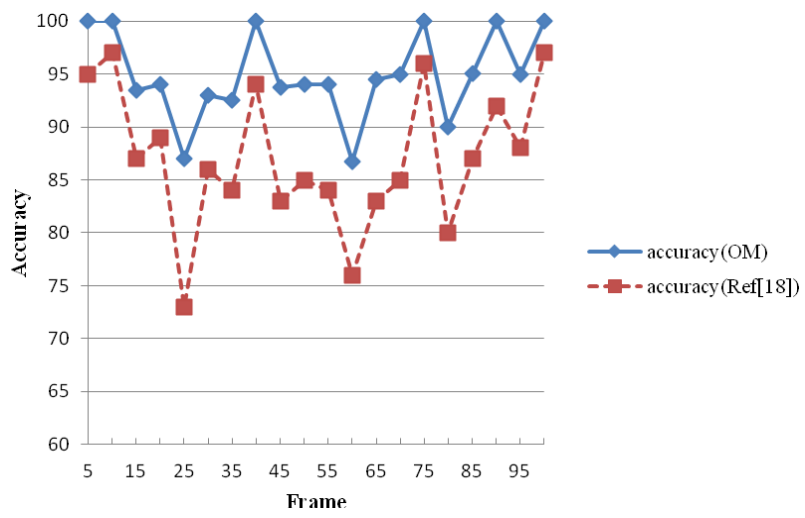

Figure 9. Comparison of recognition accuracy

As observed in Fig.8, the performance of our detection approach is satisfactory in detecting the passenger flow safety in high-speed railway transport hub. The recognition amounts in different frames are close to the actual passenger amount in surveillance video. In Fig.9, the recognition accuracy of our methods is significantly improved for the Ref [18]. After a great deal of experiments, the average accuracy of passenger flow safety detection based on our algorithm can reach $94.9 \%$, and is better than the average accuracy of Ref [18]. The accurate passenger flow safety detection is significant for the safety management of high-speed railway transport hub.

\section{CONCLUSION}

In this paper, we considered the passenger flow safety detection in high-speed railway transport hub. According to the sensor-based detection process for passenger flow safety, an improved watershed algorithm based on tag obtained automatically was proposed for precise image segmentation, and a recognition algorithm based on connected domain characters was proposed to accurately recognize the passenger flow safety. Computational experiments on sensor data from a specific high-speed railway transport hub showed that the proposed approach was effective and efficient in detecting the passenger flow safety. The detection adaptability for different passenger flow density is a possibility for future research.

\section{ACKNOWLEDGMENTS}

This research was supported by the Fundamental Research Funds for the Central Universities (Grant no. 2015JBM044), the Talented Faculty Funds of Beijing Jiaotong University (Grant no. 2014RC005) and State Key Laboratory Program (Grant no. RCS 2016ZT016)

\section{REFERENCES}

[1] I. Haritaoglu, D. Harwood and L Davis, "W4 : who? when? where? what? A real time system for detecting and tracking people," Proceedings of Third IEEE International Conference on Automatic Face and Gesture Recognition, pp. 222-227, 1998.

[2] B. Benfold and I.D Reid, "Stable Multi-Target Tracking in Real-Time Surveillance Video," Proceedings of IEEE Conference on Computer Vision and Pattern Recognition, pp. 3457-3464. 2011

[3] M.J Deilamani and R.N Asli, "Moving object tracking based on mean shift algorithm and features fusion," International Symposium on Artificial Intelligence and Signal Processing, pp. 48-53, 2011.

[4] M.J.V Leach, E.P Sparks and N.M Robertson, "Contextual anomaly detection in crowded surveillance scenes," Pattern Recognition Letters, vol. 44, pp. 71-79, 2014.

[5] S.H Choa and H.B Kang, "Abnormal behavior detection using hybrid agents in crowded scenes," Pattern Recognition Letters, vol. 44, pp. 6470,2014

[6] X.B Zhu, J Liu, J.Q Wang, C.S Li and H.Q Lu, "Sparse representation for robust abnormality detection in crowded scenes," Pattern Recognition, vol. 47, pp. 1791-1799, 2014.

[7] G.S.K Fung, N.H.C Yung and G.K.H Pang, "Vehicle shape approximation from motion for visual traffic surveillance," Proceedings of Intelligent Transportation Systems, pp. 608-613, 2011.

[8] H Asaidi, A Aarab and M Bellouki, "Shadow elimination and vehicles classification approaches in traffic video surveillance context," Journal of Visual Languages \& Computing, vol. 25, pp. 333-345, 2014.

[9] B Luo and J.B Xia, "A novel intrusion detection system based on feature generation with visualization strategy," Expert Systems with Applications, vol.41, pp. 4139-4147, 2014.

[10] J.L Castro, M Delgado, J Medina and M.D Ruiz-Lozano, "Intelligent surveillance system with integration of heterogeneous information for intrusion detection," Expert Systems with Applications, vol.38, pp. 11182-11192, 2011.

[11] D. Y Chen and P. C Huang, "Motion-based unusual event detection in human crowds," Journal of Visual Communication and Image Representation, vol. 22, pp. 178-186, 2011.

[12] Y Cong, J.S Yuan and J Liu, "Abnormal event detection in crowded scenes using sparse representation," Pattern Recognition, vol.46, pp. 1851-1864, 2013. 
[13] K.X Dai, G.H Li, D Tu and J Yuan, "Prospects and Current Studies on Background Subtraction Techniques for Moving Objects Detection from Surveillance Video," Journal of Image and Graphics, vol.11, pp. 919927, 2006.

[14] Z.Y Xie, B.T Dong, Y Chen and Q Li, "Comparative Study on Moving Object Detection Method in the High-speed High-speed railway transport hub Video Surveillance System," Key Engineering Materials, vol. 467-469, pp. 503-508, 2011.

[15] R Girisha and S Murali, "Segmentation of motion objects from surveillance video sequences using partial correlation," IEEE Conference on image processing, pp. 1129-1132, 2009.
[16] Z.Y Xie, L.M Jia, Y Qin and L Wang, "A hybrid Temporal-spatio Forecasting Approach for Passenger flow safety in Chinese High-speed High-speed railway transport hub," Discrete Dynamics in Nature and Society, vol. 2013, pp. 1-9, 2013.

[17] Z.Y Xie, L.M Jia, Y Qin and L Wang, "Background Modeling of Moving Object Detection in High-speed High-speed railway transport hub Video Surveillance," Journal of Convergence Information Technology, vol. 8, pp. 44-52, 2013.

[18] Z.Y Xie, L.M Jia, Y Qin and L Wang, "Passenger Flow Detection of Video Surveillance: A Case Study of High-Speed High-speed railway transport hub in China," ELEKTRONIKA IR ELEKTROTECHNIKA, vol. 21 , pp. 48-53, 2015. 